\title{
Hernie discale: toujours opérer?
}

Swiss Medical Board

\begin{abstract}
Le Swiss Medical Board a analysé dans son dernier rapport la nécessité d'une intervention chirurgicale en cas de hernie discale. Il conclut que, dans la phase aiguë, en l'absence de déficits moteurs fonctionnels, le traitement conservateur doit être privilégié par rapport à une intervention chirurgicale. Toutefois, sans résultats positifs après trois mois ou en cas d'aggravation de l'état du patient, une intervention chirurgicale doit être envisagée et le patient doit en être informé en détail.
\end{abstract}

Les douleurs lombaires sont fréquentes et leurs origines sont variées. La plupart du temps, elles sont dues à des hernies discales. En compressant l'un des nerfs spinaux à droite ou à gauche de la colonne vertébrale, les hernies discales peuvent les irriter ou les endommager (radiculopathie) avec des symptômes allant de douleurs dans la jambe correspondante jusqu'à des troubles de la sensibilité (déficits de la sensibilité) et des paralysies musculaires (déficits de la motricité).

Les radiculopathies peuvent être traitées par la méthode conservatrice ou par une intervention chirurgicale. Dans la phase précoce, on applique le plus souvent le traitement conservateur, dont l'objectif prioritaire est de soulager les douleurs. En cas de troubles légers, ce traitement est en général suffisant. En revanche, en cas de déficits moteurs fonctionnels, une intervention chirurgicale est le plus souvent indiquée. Dans de nombreuses situations, pourtant, le choix du traitement optimal est difficile. Tel est l'objet du rapport «Radiculopathies lombaires aiguës ou subaiguës dues à des hernies discales: traitement conservateur versus chirurgical».

\section{Recommandations quant à la thérapie et informations sur l'activité de recherche}

Un groupe de travail universitaire indépendant a traité des données concernant les preuves de l'efficacité médicale et les a présentées dans le rapport d'assessment. Un groupe d'experts a recueilli les prises de position des partenaires concernés (sociétés de discipline médicale, thérapeutes, bailleurs de fonds, patients) et a élaboré, sur cette base, des évaluations et des recommandations dans le rapport d'appraisal. Ce faisant, les avis exprimés lors des consultations ont été intégrés dans toute la mesure du possible dans les conclusions. Les données actuelles concernant les deux formes de thérapie sont lacunaires et dépassées. De même, qu'il s'agisse du traitement conservateur ou du traitement chirurgical, il n'existe pas de données relatives aux principaux développements. Il est d'autant plus important que les informations concernant les standards actuellement appliqués en Suisse soient prises en considération.

Le conseil des experts a formulé les recommandations suivantes, en tenant compte du rapport d'assessment et des commentaires des fournisseurs de prestation concernés:

- Les patients atteints de radiculopathies aiguës ( $<6 \mathrm{se}$ maines) ou subaiguës (<12 semaines) dues à des hernies discales, sans déficits moteurs fonctionnels doivent être traités dans le cadre d'une approche thérapeutique interdisciplinaire, tout en donnant la priorité au traitement conservateur.

- Les patients doivent être informés en détail et de manière adéquate des avantages et des inconvénients des différentes approches thérapeutiques, en particulier de la nature et de la fréquence des possibles effets indésirables.

- Face à l'insuffisance de données probantes, des efforts de recherche doivent être envisagés, financés et réalisés dans les quatre domaines suivants:

1. Une étude randomisée, d'une ampleur appropriée, destinée à comparer les approches prioritairement chirurgicales et les approches prioritairement conservatrices sur la base des modalités de traitement actuelles.

2. Des études randomisées comparatives pour l'analyse des effets désirables et indésirables de l'ensemble des traitements conservateurs.

3. Des recherches de critères de pronostic pour l'identification des groupes de patients susceptibles de profiter d'une opération à un stade précoce.

4. Une meilleure documentation des évolutions à long terme après un traitement prioritairement conservateur et après une approche prioritairement chirurgicale.

Les rapports d'assessment et d'appraisal sont publiés sur le site internet du Swiss Medical Board. 